\title{
Diagnosis of UAV Pitot Tube Defects Using Statistical Change Detection
}

\author{
Hansen, Søren; Blanke, Mogens; Adrian, Jens
}

Published in:

7. Symposium on Intelligent Autonomous Vehicles

Publication date:

2010

Document Version

Early version, also known as pre-print

Link back to DTU Orbit

Citation (APA):

Hansen, S., Blanke, M., \& Adrian, J. (2010). Diagnosis of UAV Pitot Tube Defects Using Statistical Change Detection. In 7. Symposium on Intelligent Autonomous Vehicles

\section{General rights}

Copyright and moral rights for the publications made accessible in the public portal are retained by the authors and/or other copyright owners and it is a condition of accessing publications that users recognise and abide by the legal requirements associated with these rights.

- Users may download and print one copy of any publication from the public portal for the purpose of private study or research.

- You may not further distribute the material or use it for any profit-making activity or commercial gain

- You may freely distribute the URL identifying the publication in the public portal

If you believe that this document breaches copyright please contact us providing details, and we will remove access to the work immediately and investigate your claim 


\title{
Diagnosis of UAV Pitot Tube Failure Using Statistical Change Detection
}

\author{
Søren Hansen ${ }^{*, * *}$ Mogens Blanke ${ }^{*, * *}$ Jens Adrian ${ }^{* *}$ \\ * Technical University of Denmark, Department of Electrical Engineering, \\ Automation and Control Group, Elektrovej B. 326, DK-2800 Lyngby. Denmark. \\ ** Danish Forces Joint UAV Team, Naval Weapons School, \\ Gnibenvej 55, DK-4583 Sjallands Odde. Denmark. \\ *** CeSOS, Marine Technology Centre, Norwegian University of \\ Science and Technology, NTNU, NO-7491 Trondheim, Norway. \\ (E-mail: sh@elektro.dtu.dk,mb@elektro.dtu.dk, adrian@mil.dk)
}

\begin{abstract}
Unmanned Aerial Vehicles need a large degree of tolerance to faults. One of the most important steps towards this is the ability to detect and isolate faults in sensors and actuators in real time and make remedial actions to avoid that faults develop to failure. This paper analyses the possibilities of detecting faults in the pitot tube of a small unmanned aerial vehicle, a fault that easily causes a crash if not diagnosed and handled in time. Using as redundant information the velocity measured from an onboard GPS receiver, the air-speed estimated from engine throttle and the pitot tube based airspeed, the paper analyses the properties of residuals. A dedicated change detector is suggested that works on pre-whitened residuals and a generalised likelihood ratio test is derived for a Cauchy probability density, which the residuals are observed to have. A detection scheme is obtained using a threshold that provides desired quantities of false alarm and detection probabilities. Fault detectors are build based on raw residual data and on a whitened edition of these. The two detectors are compared against recorded telemetry data of an actual event where a pitot tube defect occurred. (C)2010 IFAC
\end{abstract}

Keywords: Fault detection, Change detection, Unmanned Aerial Vehicle, Pitot tube

\section{INTRODUCTION}

Defects on sensors can have catastrophic consequences for airplanes, specially smaller airplanes, which do not have the same sensor redundancy that is available on larger aircraft. It is therefore important to be able to detect whether a sensor defect has occurred. One of the most important sensors is the pitot tube that measures airspeed of the vehicle. This sensor is very exposed because of its position in the airstream and can easy be clogged by dust or water particles that freeze at higher altitudes, when they come in contact with the airplane body.

The solution to these clogging problems usually employed on larger aircrafts is to install several pitot tubes with build in heating devices to have a redundant system that can accommodate icing. Because of weight and space limitations, adding more sensors is usually not an option on smaller unmanned aerial vehicles (UAV). Therefore, a different approach must be taken to diagnose and accommodate faults. One way could be to have artifact readings detected and replaced with estimated values. Detection of faults and fault-tolerance for UAVs has a lot of focus and, as described in Ducard (2009), many parts of the aircraft control and operation can benefit from using fault tolerant methods. A systematic approach to fault detection is described in Fravolini et al. (2009) and some of the applications of these methods are, detection of mechanical defects, like stuck control surfaces. These were studied in Bateman et al. (2009) and Park et al. (2009) where active methods were used to isolate faults. Observer based fault diagnosis was investigated in e.g. Heredia et al. (2008). Nonlinear models that describe the aircraft can also be used in fault diagnosis, this was demonstrated on small helicopters in Freddi et al. (2009).

This paper investigates the use of GPS velocity measurements and propeller thrust readings to generate speed information that is redundant compared to the pitot tube airspeed. Residual values are formed from which defects on the pitot tube can be isolated. The detection is done using statistical information gathered from telemetry records from a Banshee UAV (see fig. 1). Advantage is taken of availability of real data to derive probability density functions and establish spectral properties of data. Dedicated change detection is derived based on observed signal properties and detectors are convincingly tested on real data from an incident involving icing of the pitot tube.

\section{PLATFORM}

The UAV studied in this paper is a Banshee target drone build by the British company Meggitt Defence System.

The airplane is controlled by an autopilot and the operator sends set-point requests to height, speed and heading. Telemetry data are send from the plane and logged at a ground station. These data are the basis for the investigations presented in this paper. 
The Banshee is a delta wing aircraft equipped with a small rear mounted engine. The thrust is delivered by a twobladed wooden propeller. It is launched from a catapult system (see fig. 1) and lands by parachute. It is therefore not equipped with any landing gear.

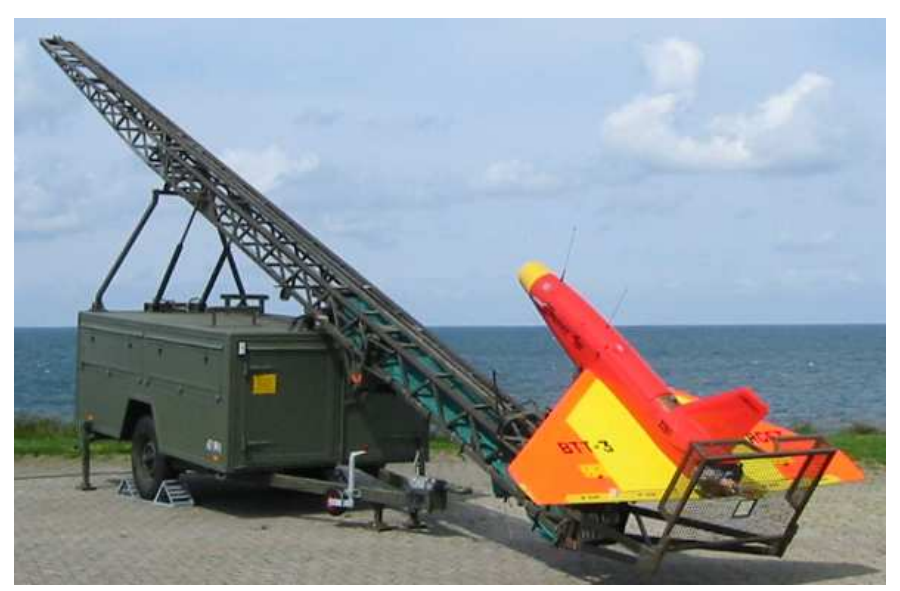

Fig. 1. Banshee UAV ready for launch at the Danish Naval Weapons School at Sjællands Odde. (Foto: VFD)

\section{MODEL}

The airplane is modelled by the dynamic and kinematic equations, which describe its motion through the air. In order to describe these concepts mathematically, a number of reference frames are needed. These are the ECEF (Earth centred earth fixed) frame, the VCE (Vehicle carried earth) frame, the body frame and the wind frame, see e.g Stevens and Lewis (2003) for details.

\subsection{Airplane motion parameters}

The airplane's motion is described by its velocities in the body coordinate system

$$
\boldsymbol{v}_{b}=[u, v, w]^{\mathrm{T}},
$$

and the rotational rate about the body axes,

$$
\boldsymbol{\omega}=[p, q, r]^{\mathrm{T}} \text {. }
$$

The Euler angles used to describe the rotation between the vehicle carried coordinate frame and the body frame of the aircraft are,

$$
\boldsymbol{\Phi}=[\phi, \theta, \psi]^{\mathrm{T}} \text {. }
$$

This is also the attitude and heading information.

\subsection{Kinematics}

The kinematics of the airplane relates the angular velocities $\boldsymbol{\omega}$ to the rate of change in the Euler angles $\dot{\boldsymbol{\Phi}}$, which are used in the rotation between body and the VCE coordinate system. This is given by the following relation

$$
\begin{aligned}
\dot{\boldsymbol{\Phi}} & =\boldsymbol{L}(\boldsymbol{\Phi}) \boldsymbol{\omega} \\
\boldsymbol{L}(\boldsymbol{\Phi}) & =\left[\begin{array}{ccc}
1 & \tan \theta \sin \phi & \tan \theta \cos \phi \\
0 & \cos \phi & -\sin \phi \\
0 & \sin \phi / \cos \theta & \cos \phi / \cos \theta
\end{array}\right]
\end{aligned}
$$

The change in position in the ECEF reference frame can be described by rotating the translational velocities $\boldsymbol{v}_{b}$ to the navigation frame. The transformation matrix involved in this depends on $\boldsymbol{\Phi}$.

\subsection{Dynamics}

The airplane is seen as a rigid body moving through the air. Newton's and Euler's laws for linear and angular momentum relate forces and moments acting on the rigid body to linear and angular velocities and accelerations. Since the UAV is flying at low speed and height over a relatively small area, we assume that the Earth is flat, and Coriolis forces and the centripetal force from the curvature of the Earth can be neglected.

The airplane is affected by a thrust from the engine $\boldsymbol{F}_{T}$, an aerodynamic force $\boldsymbol{F}_{A}$ arising from the lift and drag from the airplane body and, naturally, the gravitational force from Earth $\boldsymbol{F}_{G}$. With mass $m$,

$$
\frac{d}{d t}\left(m \boldsymbol{v}_{b}\right)=\sum \boldsymbol{F}=\boldsymbol{F}_{T}+\boldsymbol{F}_{A}+\boldsymbol{F}_{G}
$$

Since this equation is expressed in the inertial frame (the Earth system) the equation of Coriolis is used to find the derivative,

$$
m \dot{\boldsymbol{v}}_{b}+\boldsymbol{\omega} \times m \boldsymbol{v}_{b}=\boldsymbol{F}_{T}+\boldsymbol{F}_{A}+\boldsymbol{F}_{G}
$$

For an in depth description of airplane dynamics see for instance Stevens and Lewis (2003).

\subsection{Thrust}

The Banshees' thrust is delivered by a rear mounted internal combustion engine that drives a 2 bladed wood propeller. The actual force delivered depends on the physical dimensions of the propeller, the engines revolutions and the density of the air. Given a propeller with diameter $d_{p}$ rotating at $n$ revolutions per second the thrust force is given by:

$$
F_{T}=k_{T} \varrho n^{2} d_{p}^{4}
$$

where $k_{T}$ is the thrust coefficient and $\varrho$ is the density of air. Wind tunnel tests (see Lesley (1939)) of propellers of the used type show that the thrust coefficient is approximately linear with the advance ratio $J$ as seen in Lesley (1939) figure 5 . The advance ratio is given by:

$$
J=\frac{v}{n d_{p}}
$$

In nominal flight the advancement ratio is in the range 0.9 to 1.1 .

Propellers are usually characterised by their diameter $d_{p}$ and pitch $P_{p}$. These values can be combined to a nondimensional pitch given by:

$$
P^{\prime}=\frac{P_{p}}{d_{p}}
$$

In Nissen (2002) the following scaling for the thrust coefficient was proposed.

$$
k_{T}(J) \simeq k_{T, \text { ref }}\left(J-\left(P^{\prime}-P_{\text {ref }}^{\prime}\right)\right)
$$

where $k_{T \text {,ref }}$ and $P_{\text {ref }}^{\prime}$ is the thrust coefficient and nondimensional pitch of the reference signal respectively. The reference signals originates from different propellers tested in Lesley (1939). Since the $26 \times 30$ inch propeller used on the Banshee has $P^{\prime}$, which is equal to one of the tested propellers, the characteristics of this propeller is used from 
this point on.

The thrust coefficient can be expressed in two terms describing the linear area seen in figure 5 in Lesley (1939).

$$
k_{T}=k_{T 0}+k_{T 1} J
$$

By calculating the lift force of the propeller it was shown in Blanke (1981) that the thrust can be expressed by:

$$
\begin{aligned}
F_{T} & =T_{n n} n^{2}+T_{n u} n u \\
T_{n n} & =k_{T 0} \varrho d^{4} \\
T_{n u} & =k_{T 1} \varrho d^{3}
\end{aligned}
$$

The thrust force works in the $x$-direction of the body system. This gives rise to the following force equation in the $x$-direction by using Equation (7):

$$
m \dot{u}+m(q w-r v)=F_{T x}+F_{A x}+m g \sin \theta
$$

The aerodynamic force $F_{A x}$ is a combination of lift and drag forces on the airplane body. Since the angle of attack $\alpha$ is usually small this force primarily consists of the drag on the airplane. This is modelled on basis of recorded data to vary with $\theta$. It is assumed that the engine is aligned with the airplane body and therefore $F_{T x}=F_{T}$. In straight and level flight with no accelerations present we can estimate the forward air speed of the plane by inserting the thrust expression from equation 13.

$$
\hat{u}=\frac{-T_{n n} n^{2}-F_{A x}-m g \sin \theta}{T_{n u} n}
$$

While this simple estimate was found to suffice for the purpose of fault diagnosis, more elaborate and sophisticated nonlinear observers and estimators have been suggested in the literature. Zhou and Blanke (1989) described a way to estimate state and parameters in nonlinear systems with a structure similar to the thrust equation here, Blanke et al. (1998) applied an adaptive observer scheme and Pivano et al. (2009) showed nonlinear observer designs for thrust estimation.

\section{SIGNAL ANALYSIS}

The airspeed of the UAV is measured by pressure differences between the pitot tube input and the static vent input. This gives the difference between the stagnation pressure and the static pressure which, according to Bernoulli's theorem, is the dynamic pressure $\bar{q}$,

$$
\bar{q}=\frac{1}{2} \varrho V^{2}
$$

From this the planes airspeed $V$ can be calculated.

In order to detect an error in the airspeed indicator this sensor value must be compared to similar measurements. The GPS receiver gives a speed from its internal Kalman filter. This corresponds to the speed in an ECEF reference frame, but using knowledge about the wind speed and direction it can be transformed to airspeed measured in the pitot tube.

As seen in the previous chapter the speed in the body $\mathrm{x}$-direction can be derived from the thrust equations developed by the engine, by using equation 17 . This measure can also be converted to match the airspeed measured by the pitot tube system.

The estimation of the wind direction and wind speed are crucial for the correctness of these conversions. These values are also hard to estimate with common means. However the wind is measured at the ground station, and also estimated during takeoff where the airplane always climbs into the wind direction. These estimates prove to give good results in practice.

The simplest way of comparing the three airspeed measurements are by subtracting them from each other. This creates three residual signals which in the nominal case should have a values around 0 . If we denote the airspeed measured by the pitot tube $v_{\text {airspeed }}$, the airspeed calculated from the GPS $v_{\text {gps }}$ and the airspeed calculated from the engine velocity $v_{\text {thrust }}$ the following parity matrix can be formed:

\begin{tabular}{c|ccc} 
& $v_{\text {airspeed }}$ & $v_{\text {gps }}$ & $v_{\text {thrust }}$ \\
\hline$R_{1}$ & $s$ & $s$ & 0 \\
$R_{2}$ & $s$ & 0 & $s$ \\
$R_{3}$ & 0 & $s$ & $s$
\end{tabular}

An 's' in list 19 means that errors on this value is strongly detectable by the given residual. All the residual are generated from the difference between measurements of the airspeed velocity. This means that if the residual is different from zero it indicates a fault, on one of the signals. Therefore an detection of a DC-signal of unknown magnitude, different from zero, in the residual, would indicate an error.

Since only $R_{1}$ and $R_{2}$ contains pitot pressure information only these two residuals are analysed onwards, however the same methods are applied to $R_{3}$ to obtain fault isolation.
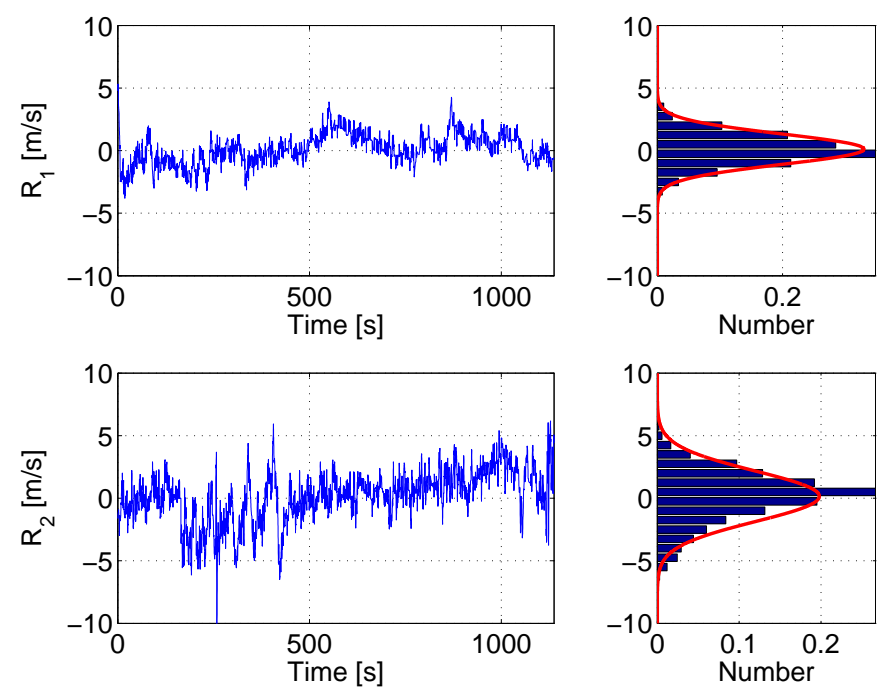

Fig. 2. Time development and histogram for residual $R_{1}$ and $R_{2}$ in the fault free case.

Figure 2 shows a time history and a histogram for $R_{1}$ and $R_{2}$ in the fault free case.

As seen from the power spectrum densities in figure 3 the noise present at the residual signals are not white. Since white noise is one of the requirements for most statistical change detectors to perform optimal, the lowpass filtered nature of the noise should be removed. One solution of dealing with coloured noise is to filter white noise through a suitable filter function to take into account the correlations present in the coloured noise. This can be created from a large record of data where all the signals 


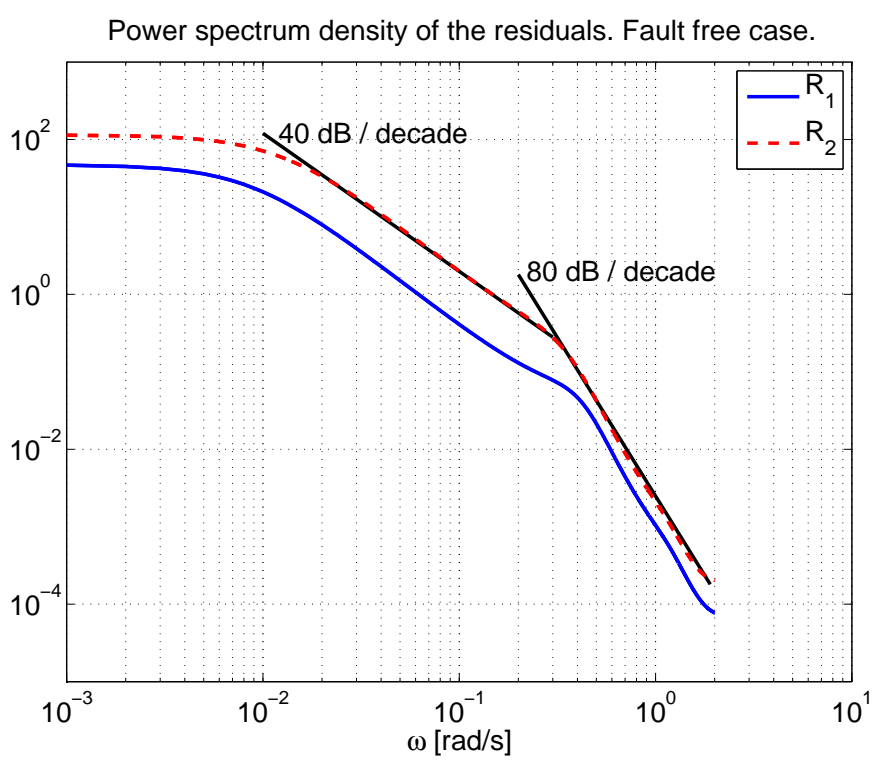

Fig. 3. Power spectrum densities for residual $R_{1}$ and $R_{2}$. properties are present. As indicated on figure 3 the power spectrum density of the two residuals consists of a part which decreases with $40 \mathrm{~dB} /$ decade and a part which decreases with $80 \mathrm{~dB} /$ decade.

A filter function $H$ which converts a white noise input to a coloured noise output according to

$$
S_{r r}(\omega)=|H(\omega)|^{2} S_{w w}(\omega)=H(j \omega) H^{*}(j \omega) S_{w w}(\omega)
$$

can be created. The function should satisfy the shape given in figure 3 and satisfy that

$$
\frac{1}{2 \pi} \int_{-\infty}^{\infty} S_{r r}(\omega) d \omega=\sigma_{r}^{2}
$$

where $\sigma_{r}^{2}$ is the variance of the residual noise. In order to satisfy the shape in the following PSD function is chosen.

$$
\mathcal{P}(\omega)=K \frac{\tau_{1} \omega^{2}+1}{\left(\tau_{1} \omega^{2}+1\right)^{2}} \frac{\tau_{2} \omega^{2}+1}{\left(\tau_{2} \omega^{2}+1\right)^{2}}
$$

where $\tau_{1}$ is the reciprocal cut-off frequency between the $0 \mathrm{~dB} /$ decade and the $-40 \mathrm{~dB} /$ decade parts and $\tau_{2}$ is the time constant between $-40 \mathrm{~dB} /$ decade and $-80 \mathrm{~dB} /$ decade parts, and $K$ is the magnitude. This corresponds to the following filter function.

$$
\begin{aligned}
& \mathcal{P}(\omega)=H(j \omega) H^{*}(j \omega)= \\
& \sqrt{K} \frac{\sqrt{\tau_{1}} j \omega+1}{\left(\sqrt{\tau_{1}} j \omega+1\right)^{2}} \frac{\sqrt{\tau_{2}} j \omega+1}{\left(\sqrt{\tau_{2}} j \omega+1\right)^{2}} \times \\
& \sqrt{K} \frac{-\sqrt{\tau_{1}} j \omega+1}{\left(-\sqrt{\tau_{1}} j \omega+1\right)^{2}} \frac{-\sqrt{\tau_{2}} j \omega+1}{\left(-\sqrt{\tau_{2}} j \omega+1\right)^{2}}
\end{aligned}
$$

The constants in the equation are found by optimising $H(j \omega)$ to fit the actual spectra.

Expressing the filter function function in terms of $s$ gives

$$
H(s)=\sqrt{K} \frac{\sqrt{\tau_{1}} s+1}{\left(\sqrt{\tau_{1}} s+1\right)^{2}} \frac{\sqrt{\tau_{2}} s+1}{\left(\sqrt{\tau_{2}} s+1\right)^{2}}
$$

The filter is implemented digitally using an IIR representation. Since filtering white noise through $H(s)$ should create a noise signal with a power spectrum similar to the one in figure 3 filtering $R_{1}$ and $R_{2}$ through $H^{-1}(s)$ should create a signal with a flat power spectrum. Figure 5
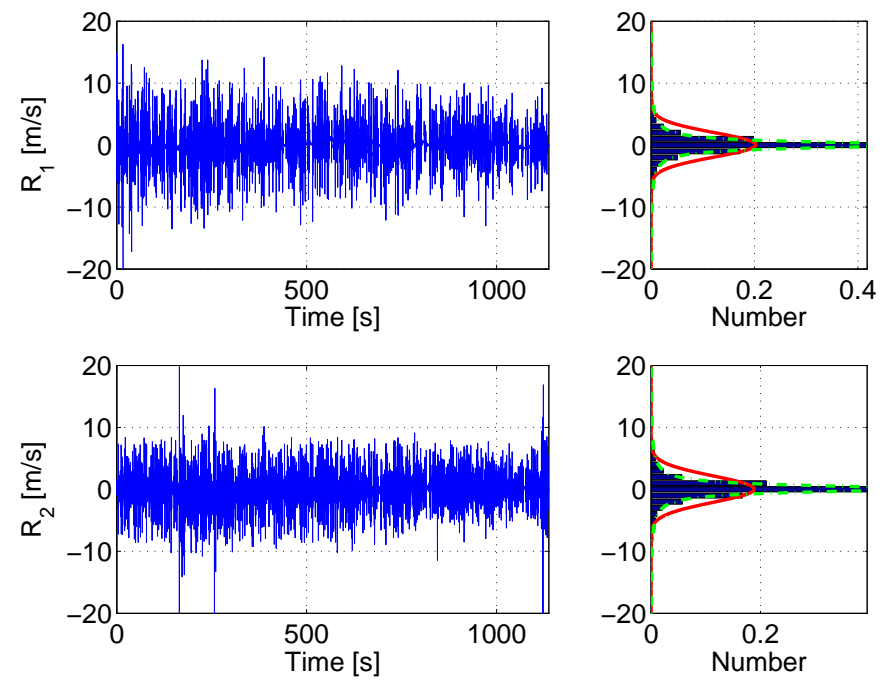

Fig. 4. Time development and histogram for the whitened residual $R_{1}$ and $R_{2}$ in the fault free case.
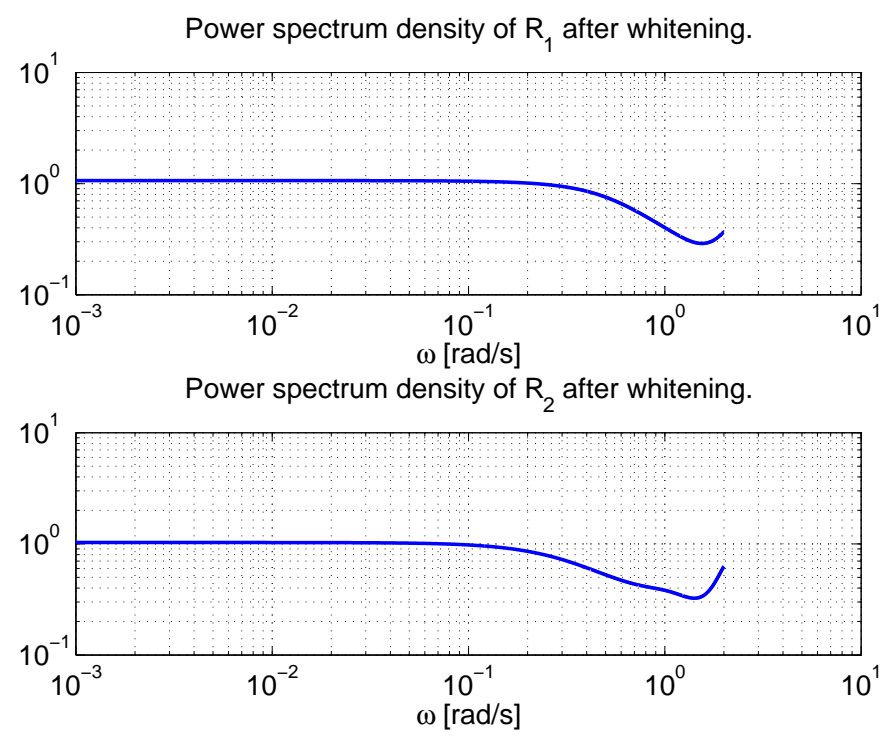

Fig. 5. Power spectrum densities after whitening for residual $R_{1}$ and $R_{2}$ in the fault free case.

shows the result of the whitening. Both residuals features now white noise. In figure 4 the time development and the histogram is plotted. Neither of the residuals are now Gaussian distributed. Instead they follow the Cauchy distribution (equation 34) very well as indicated by the green dotted line in the histogram. The Gaussian nature that the residuals has before whitening comes from the effect of the low-pass filtering, which creates the power spectra observed. When this is removed during the whitening process the Gaussianity is also removed.

\section{DETECTORS}

Two approaches to change detection is investigated. In the first one the raw residual signals are used and the next one the whitened signals are used. 


\subsection{Raw residual detector}

If the vocabulary from statistical change detection is used the scenario that it is desirable to analyse is: Detect an unknown DC-level in Gaussian white noise. If the signal is denoted $x$ we wish to distinguish between the following two hypotheses:

$$
\begin{aligned}
& \mathcal{H}_{0}: x[n]=w[n] \\
& \mathcal{H}_{1}: x[n]=A+w[n]
\end{aligned}
$$

The unknown parameters are: The DC-level $A$ and the variance of the noise $\sigma_{w}^{2}$. The GLRT (Generalised Likelihood Ratio Test) for a linear model formulation can be used to solve such a problem. However, GLRT algorithm assumes that the signal noise is white. As emphasised, this is not the case for the raw residual. However, the algorithm is tested anyway using the raw residual, to see how it performs. The general GLRT detector is given by

$$
L_{G}(\boldsymbol{x})=\frac{p\left(\boldsymbol{x} ; \hat{\boldsymbol{\Theta}}_{1}\right)}{p\left(\boldsymbol{x} ; \hat{\boldsymbol{\Theta}}_{0}\right)}>\gamma
$$

If the ration is larger than the threshold $\gamma$ this decides $\mathcal{H}_{1} . \hat{\boldsymbol{\Theta}}_{i}$ is the maximum likelihood estimate (MLE) of the parameters when hypothesis $\mathcal{H}_{i}$ is true. In general the GLRT test for the following two hypotheses:

$$
\begin{array}{ll}
\mathcal{H}_{0}: \boldsymbol{A} \boldsymbol{\Theta}=\boldsymbol{b}, & \sigma_{w}>0 \\
\mathcal{H}_{1}: \boldsymbol{A} \boldsymbol{\Theta} \neq \boldsymbol{b}, & \sigma_{w}>0
\end{array}
$$

The linear model is given by $\boldsymbol{x}=\boldsymbol{H} \Theta+\boldsymbol{w}$. The problem given by equation 25 can be transformed to this problem if we choose $\boldsymbol{\Theta}=[A], \boldsymbol{A}=[1]$ and $\boldsymbol{b}=[0]$. The matrix $\boldsymbol{H}$ is chosen to be a column of 1 corresponding to the window size $N$.

$$
T(\boldsymbol{x})=(N-1) \frac{\left(\boldsymbol{A} \hat{\Theta}_{1}-\boldsymbol{b}\right)^{\mathrm{T}}\left(\boldsymbol{A}\left(\boldsymbol{H}^{\mathrm{T}} \boldsymbol{H}\right)^{-1} \boldsymbol{A}^{\mathrm{T}}\right)^{-1}\left(\boldsymbol{A} \hat{\Theta}_{1}-\boldsymbol{b}\right)}{\boldsymbol{x}^{\mathrm{T}}\left(\boldsymbol{I}-\boldsymbol{H}\left(\boldsymbol{H}^{\mathrm{T}} \boldsymbol{H}\right)^{-1} \boldsymbol{H}^{\mathrm{T}}\right) \boldsymbol{x}}
$$

Inserting the values of $\boldsymbol{A}, \boldsymbol{b}, \boldsymbol{H}$ and $\hat{\boldsymbol{\Theta}}_{1}$ gives

$$
T(\boldsymbol{x})=(N-1) \frac{\bar{x}^{2}}{{\hat{\sigma_{w}}}^{2}}
$$

Where $\bar{x}$ is the sample mean and the MLE of the variance is given by:

$$
{\hat{\sigma_{w}}}^{2}=\frac{1}{N} \boldsymbol{x}^{\top}\left(\boldsymbol{I}-\boldsymbol{H}\left(\boldsymbol{H}^{\top} \boldsymbol{H}\right)^{-1} \boldsymbol{H}^{\top}\right) \boldsymbol{x}
$$

which just ends up with being the variance of the test data. The final test statistic is therefore

$$
T(\boldsymbol{x})=(N-1) \frac{\bar{x}^{2}}{\operatorname{var}(\boldsymbol{x})}>\gamma^{\prime}
$$

A more elaborate derivation of the detector can be found in Kay (1998). The probability of false alarms $P_{F A}$ and the probability of detection $P_{D}$ is given by:

$$
P_{F A}=Q_{F_{r, N-p}}\left(\gamma^{\prime}\right)
$$

and

$$
P_{D}=Q_{F_{r, N-p}^{\prime}(\varsigma)}\left(\gamma^{\prime}\right) ; \varsigma=\frac{N A^{2}}{\sigma_{w}^{2}}
$$

where $Q$ denotes the right tail probability.

\subsection{Pre-whitened residual detector}

The second detector works on the whitened residuals. Since these are distributed according to a Cauchy distribution (as seen from figure 4) the following probability distribution function most be used

$$
p\left(x ; x_{o}, \beta\right)=\frac{\beta}{\pi\left(x-x_{o}\right)^{2}+\beta^{2}}
$$

The two parameters is the half-width half-maximum scaling, $\beta$, and the offset $x_{o}$. The hypotheses are the same as in the nonwhite case, is a DC-level present in the noise or not. The GLRT test statistic becomes

$$
L_{G}(\boldsymbol{x})=\frac{\prod_{i=1}^{N} p\left(x_{i} ; \hat{x_{o}}, \hat{\beta}\right)}{\prod_{i=1}^{N} p\left(x_{i} ; 0, \hat{\beta}\right)}>\gamma
$$

The MLEs of $\hat{\beta}$ and $\hat{x_{o}}$ is found by fitting the data to equation 34 .

The performance can be calculated using these formulas:

and

$$
P_{F A}=Q_{\chi^{2}}\left(\gamma^{\prime}\right)
$$

$$
P_{D}=Q_{\chi^{2}(\lambda)}\left(\gamma^{\prime}\right) ; \lambda=\frac{N A^{2}}{\sigma_{w}^{2}}
$$
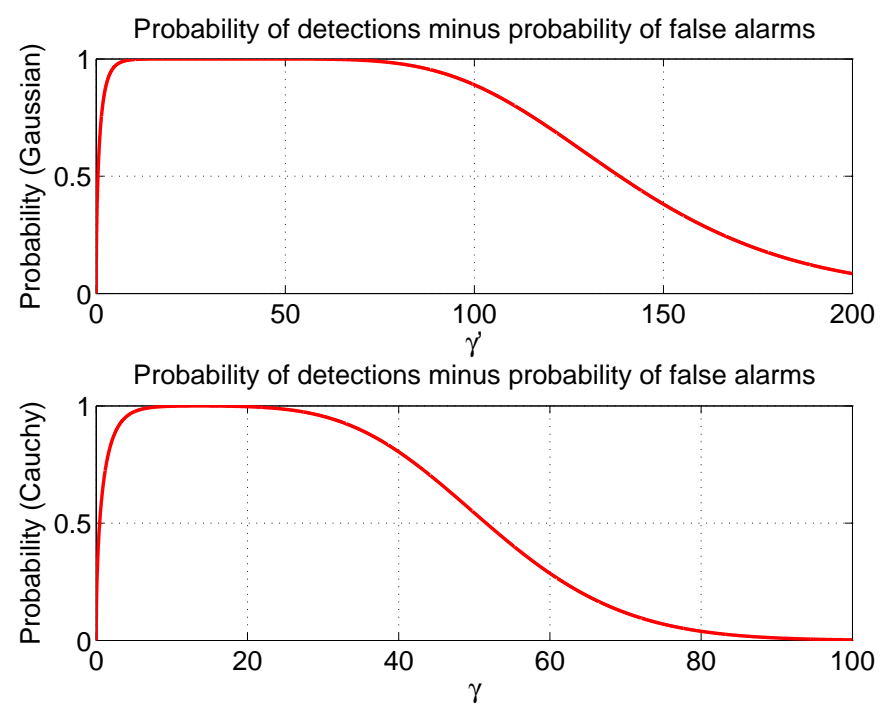

Fig. 6. Performance of the two detectors plotted as the difference between probability of detections and false alarms. Note that the value of $\gamma$ and $\gamma^{\prime}$ can not be compared numerically.

Using the performance equations the compare value $\gamma$ can be found. A plot of the detection performance minus the probability of false alarms for both the detector using the raw residual values and the one working on the whitened residuals are shown. For both performance curves a DClevel of $5 \mathrm{~m} / \mathrm{s}$ has been chosen as the value wanted to be detected. Difference below this can be down to inaccuracies in the estimation of parameters in the velocity calculations.

\section{RESULTS}

To test the two detectors, they were exposed to a data record containing a pitot tube defect. The results are shown together with the residuals in Figures 7 and 8 . 

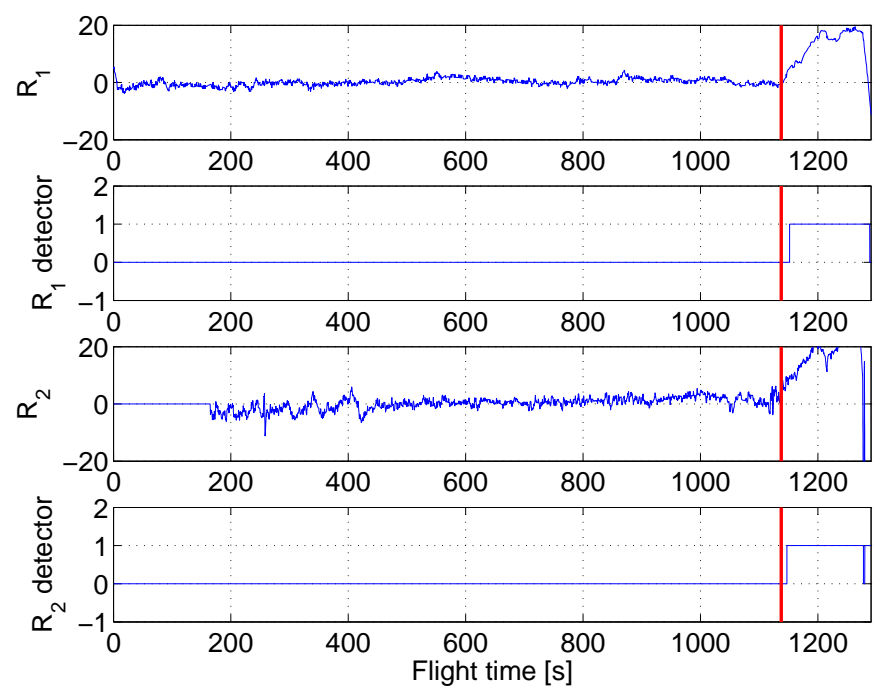

Fig. 7. Residuals and detector output. At approximately $t=1140 \mathrm{~s}$ the clogging of the pitot tube occurs (marked with the red vertical line).
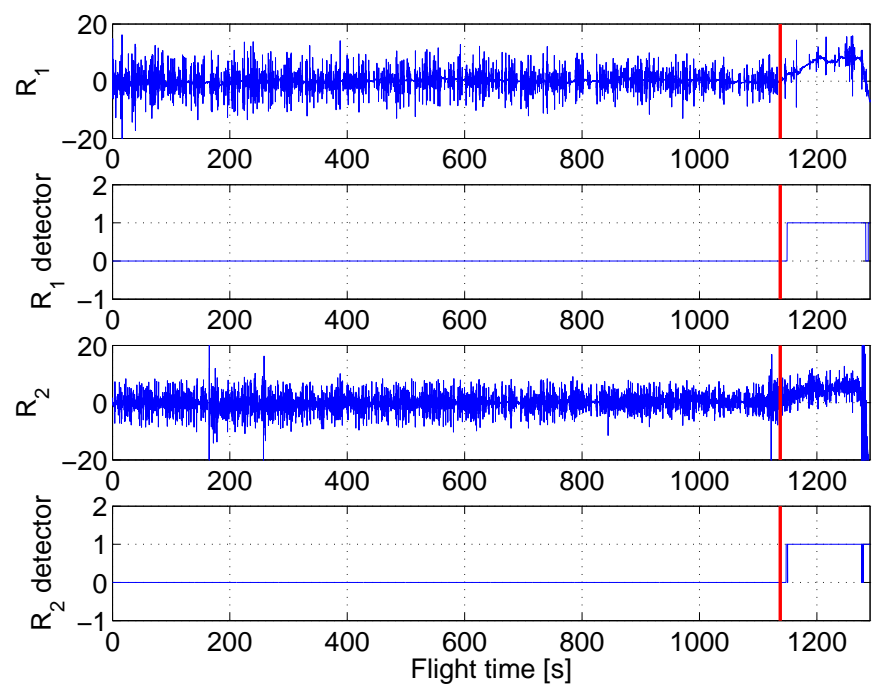

Fig. 8. Residuals and detector output. At approximately $t=1140 \mathrm{~s}$ the clogging of the pitot tube occurs (marked with the red vertical line).

As seen on the figures both detectors perform very well with the given data. They raise an alarm, indicated by a value of 1 , about 14 seconds after the incident occurs. In order to get the raw residual detector to perform as shown in figure 7 the comparison value $\gamma^{\prime}$ must be chosen 100 times larger than the one shown as maximal value in the top plot of figure 6 . The reason for this is the coloured noise degrading the performance.

\section{CONCLUSION}

The UAV's airspeed sensor faults were detected in this paper using a setup where inherent redundancies were used to generate residuals. Information from an onboard GPS receiver and the air speed estimated from engine thrust we used as sources of redundant information. Statistical properties of residuals were investigated and a whitening filter was designed to pre-whiten the residuals before applying a change detector. A dedicated detector was derived as a generalised likelihood ratio test for the log-likelihood ratio of probability density functions with and without faults. Change detection was obtained with thresholds calculated for the Cauchy distribution that pre-whitened residuals were found to follow. Detection thresholds were determined to find an optimal balance between probabilities for correct detection and false alarms. Two detectors were designed and tested against data records from an UAV with a pitot tube defect that developed while the UAV was in the air. Both detectors were able to detect the fault within an acceptable time frame and demonstrated that a hard landing could have been avoided with the proposed diagnostic system.

\section{REFERENCES}

Bateman, F., Noura, H., and Ouladsine, M. (2009). Fault tolerant control strategy based on the doa: Application to uav. In 7th IFAC Symposium on Fault Detection Supervision and Safety of Technical Processes.

Blanke, M. (1981). Ship propulsion Losses Related To Automatic Steering And Prime Mover Control. Ph.D. thesis, Technical University of Denmark.

Blanke, M., Izadi-Zamanabadi, R., and Lootsma, T. (1998). Fault monitoring and re-configurable control for a ship propulsion plant. Journal of Adaptive Control and Signal Processing, 12, 671-688.

Ducard, G.J.J. (2009). Fault-tolerant Flight Control and Guidance Systems. Springer Verlag.

Fravolini, M.L., Brunori, V., Campa, G., Napolitano, M.R., and Cava, M.L. (2009). Structured analysis approach for the generation of structured residuals for aircraft fdi. IEEE Transactions on Aerospace and Electronic Systems, 45 (4), 1466-1482.

Freddi, A., Longhi, S., and Monteriu, A. (2009). A model-based fault diagnosis system for unmanned aerial vehicles. In 7th IFAC Symposium on Fault Detection, Supervision and Safety of Technical Processes.

Heredia, G., Ollero, A., Bejar, M., and Mahtani, R. (2008). Sensor and actuator fault detection in small autonomous helicopters. Mechatronics, 18, 90-99.

Kay, S.M. (1998). Fundamentals of Statistical Signal Processing: Detection Theory. Prentice-Hall PTR.

Lesley, E.P. (1939). Propeller tests to determine the effect of number of blades at two typical solidities. Technical report, National Advisory Committee For Aeronautics.

Nissen, H.D. (2002). Instrumentation and Control of Unmanned Air Vehicle. Ph.D. thesis, Technical University of Denmark.

Park, W., Lee, S.H., and Song, J. (2009). Fault detection and isolation of durumi-ii using similarity measure. Journal of Mechanical Science and Technology, 23, 302310.

Pivano, L., Johansen, T.A., and Smogeli, O.N. (2009). A four-quadrant thrust estimation scheme for marine propellers: Theory and experiments. IEEE Transactions on Control Systems Technology, 17 (1), 215-226.

Stevens, B.L. and Lewis, F.L. (2003). Aircraft Control and Simulation. John Wiley \& Sons, 2nd edition.

Zhou, W.-w. and Blanke, M. (1989). Identification of a class of non-linear state space models using rpe techniques. IEEE Transactions of Automatic Control, 34 (3), 312-316. 